\title{
Avaliação do tipo de alimentação e relato da presença de dor em pacientes em tratamento para a mucosite oral com polihexanida.
}

\author{
Ágda Braga Teixeira ${ }^{1}$; Márcio Campos de Oliveira ${ }^{2}$; Ana Paula Eufrázio do \\ Nascimento Andrade ${ }^{3}$; Vinicius Silva Bastos de Jesus ${ }^{4}$ \\ 1. Bolsista PROBIC/CNPq, Graduando Odontologia, Universidade Estadual de Feira de Santana, e-mail: \\ agda_braga@hotmail.com \\ 2. Professor do Curso de Odontologia, Departamento de Saúde, Universidade Estadual de Feira de Santana, \\ email: \\ marciopatologia@gmail.com \\ 3. Participante do Núcleo de Pesquisa em Câncer Oral, Cirurgiã-Dentista e Mestre em Saúde Coletiva pela \\ Universidade Estadual de Feira de Santana, e-mail: \\ apeufrazio@gmail.com \\ 4. Participante Núcleo de Pesquisa em Câncer Oral, Graduando Odontologia, pela Universidade Estadual de \\ Feira de Santana, e-mail: \\ viniumbivis@gmail.com
}

\section{PALAVRAS-CHAVE: mucosite oral, ingestão de alimentos, dor. INTRODUÇÃO:}

As neoplasias malignas cada vez mais vem acometendo em larga escala a população. O câncer faz parte do grupo de doenças crônico-degenerativas que vem ocorrendo em larga escala, alterando o perfil de morbimortalidade da população brasileira. Dentre os vários tipos de câncer estão os que acometem a área de cabeça e pescoço, cujo tratamento envolve a remoção cirúrgica, radioterapia e quimioterapia. A toxicidade decorrente do tratamento oncológico em região de cabeça e pescoço acomete fortemente a mucosa oral com a morte das células epiteliais e perda da capacidade de regeneração celular natural, determinando a evolução clínica da mucosite oral (MO), que em estágios mais avançados provoca dor e dificuldade de alimentação, podendo levar até mesmo à interrupção do tratamento oncológico. Destarte, preocupa ainda não haver um protocolo de tratamento estabelecido para a MO. O presente estudo, pioneiro, visa avaliar a ingestão alimentar e relato da presença de dor em pacientes com câncer de cabeça e pescoço, com mucosite oral, antes, durante e após o tratamento com polihexanida.

\section{METODOLOGIA:}

Estudo clínico randomizado, duplo-cego, realizado com pacientes com câncer de cabeça e pescoço atendidos na Unidade de Alta Complexidade em Oncologia (UNACON), Feira de Santana - BA, no período de 2015 a 2016; com diagnóstico de MO, que assinaram o Termo de Consentimento Livre e Esclarecido e respeitaram o protocolo de adesão ao estudo. Pesquisa aprovada pelo Comitê de Ética em Pesquisa sob parecer $n^{\circ}$ 1.074.479/2015. RESULTADOS: A pesquisa abrangeu 34 participantes, sendo 17 do grupo de intervenção com gel de polihexanida (Grupo PHMB) e 17 do grupo controle (gel convencional). A idade da amostra variou de 19 a 85 anos. A maioria $(58,8 \%)$ foi do sexo masculino. Os tipos de tumores mais frequentes foram os de Orofaringe e de Cavidade Oral. Na primeira avaliação, as medianas da dor foram de 5,5 e 10 no grupo PHMB e grupo controle, com valores que iam de 0 a 10 , e de 5 a 10, respectivamente. Observava-se então que no grupo controle a intensidade da dor entre os participantes era maior (Figura 1). Entretanto, mesmo com essa vantagem no grupo PMHB com o subsequente uso do gel, ao oitavo dia, o grupo PHMB teve uma queda de mediana para 1, ou seja, 4,5 de redução de nota de dor. Resultados bons foram também observados com o grupo controle, com a mediana reduzindo para o valor 5 (figura 2). 
Os efeitos observados ao décimo quinto dia de uso do gel (figura 3 ) se mostraram muito satisfatórios, com a mediana da dor caindo para o valor 0 no grupo PHMB, enquanto no grupo controle houve um aumento da mediana para o valor 8 . A frequência da alimentação apresentou um aumento em $25 \%$ dos casos, $75 \%$ se manteve igual, e nenhum dos participantes do grupo PHMB apresentou diminuição durante a pesquisa. Da mesma maneira, a quantidade da alimentação aumentou no grupo PHMB em $75 \%$ dos participantes, considerada significativa quando comparada ao grupo controle que foi apenas de $6,7 \%$, e a diminuição neste mesmo grupo foi de 33,3\%. Dos participantes do grupo controle, $20 \%$ passaram a usar sonda para se alimentar durante a pesquisa, o que não aconteceu com nenhum integrante do grupo PHMB. Importante ressaltar que não foram relatados efeitos adversos durante o tempo de uso da substância. CONCLUSÕES: Estas primeiras evidências permitem sugerir que a polihexanida pode representar uma alternativa no controle da dor decorrente da MO, prevenindo maiores dificuldades na alimentação, o que torna o paciente menos vulnerável a suspensão do tratamento oncológico. Merece, pois, ser mais investigada, ao passo que apesar dos bons resultados obtidos, o estudo abrangeu uma quantidade limitada de pessoas, aliado à inexistência de trabalhos similares na literatura.

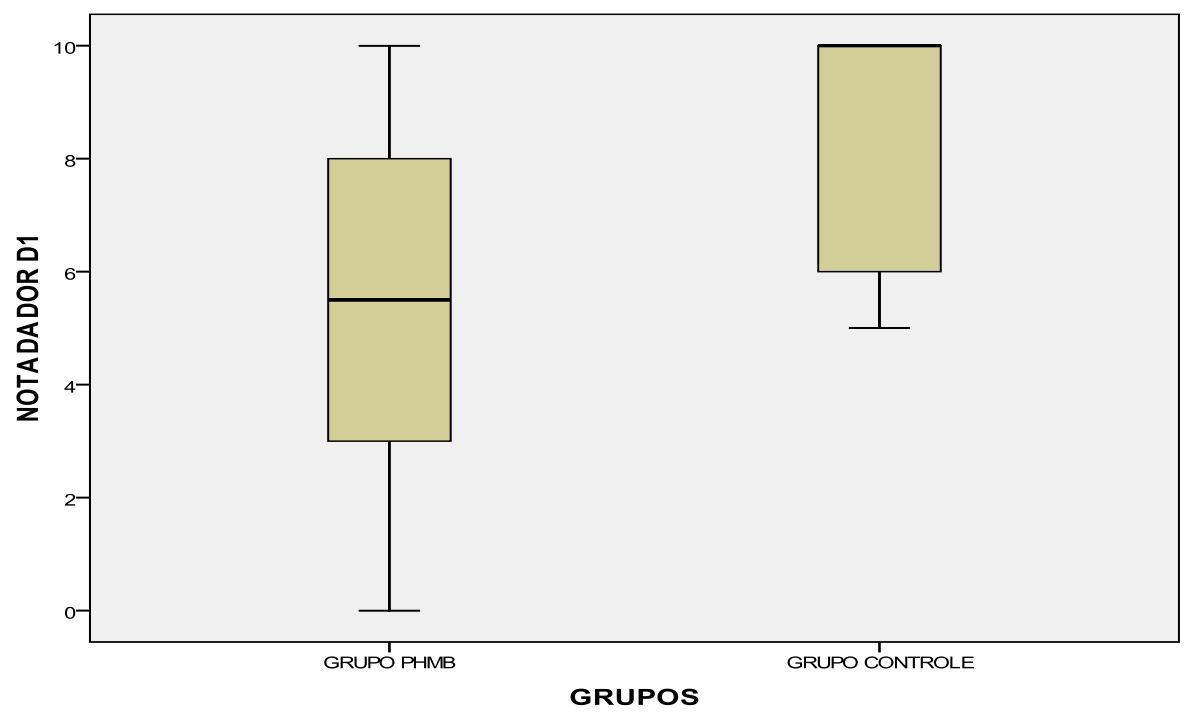

Figura 1. Distribuição da variação da média de nota de dor no D1 (10 dia de uso do gel), segundo os grupos PHMB e controle. 


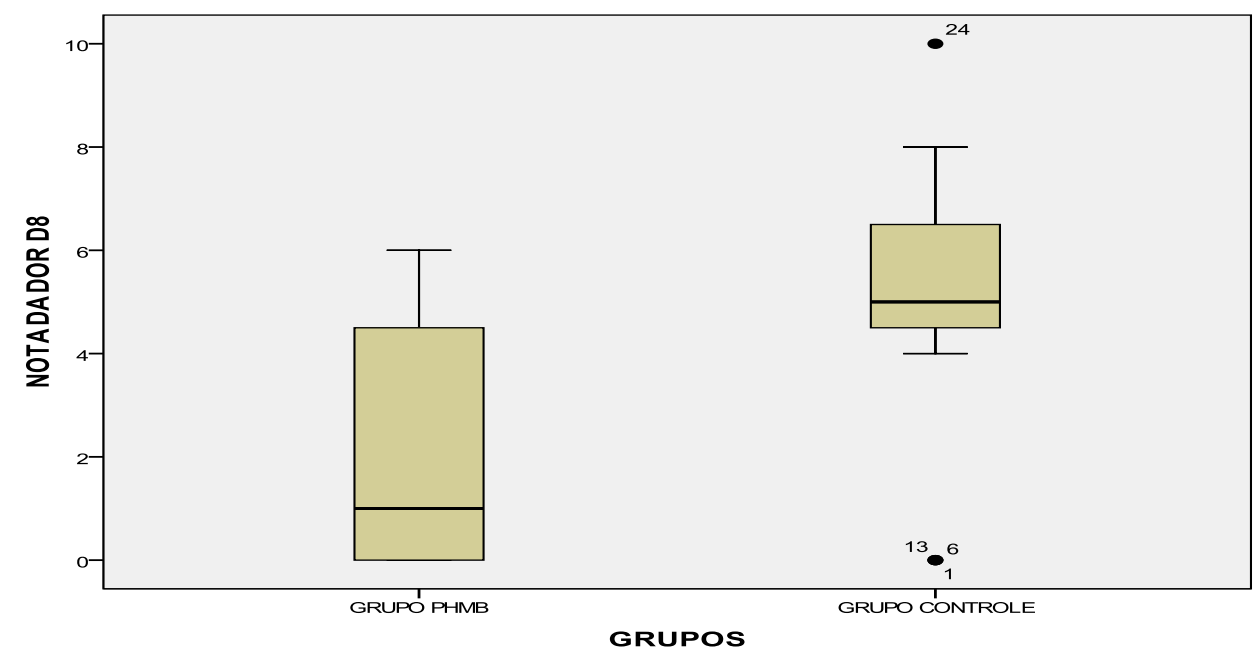

Figura 2. Distribuição da variação da média de nota de dor no D8 ( $8^{\mathrm{a}}$ dia de uso do gel), segundo os grupos PHMB e controle.

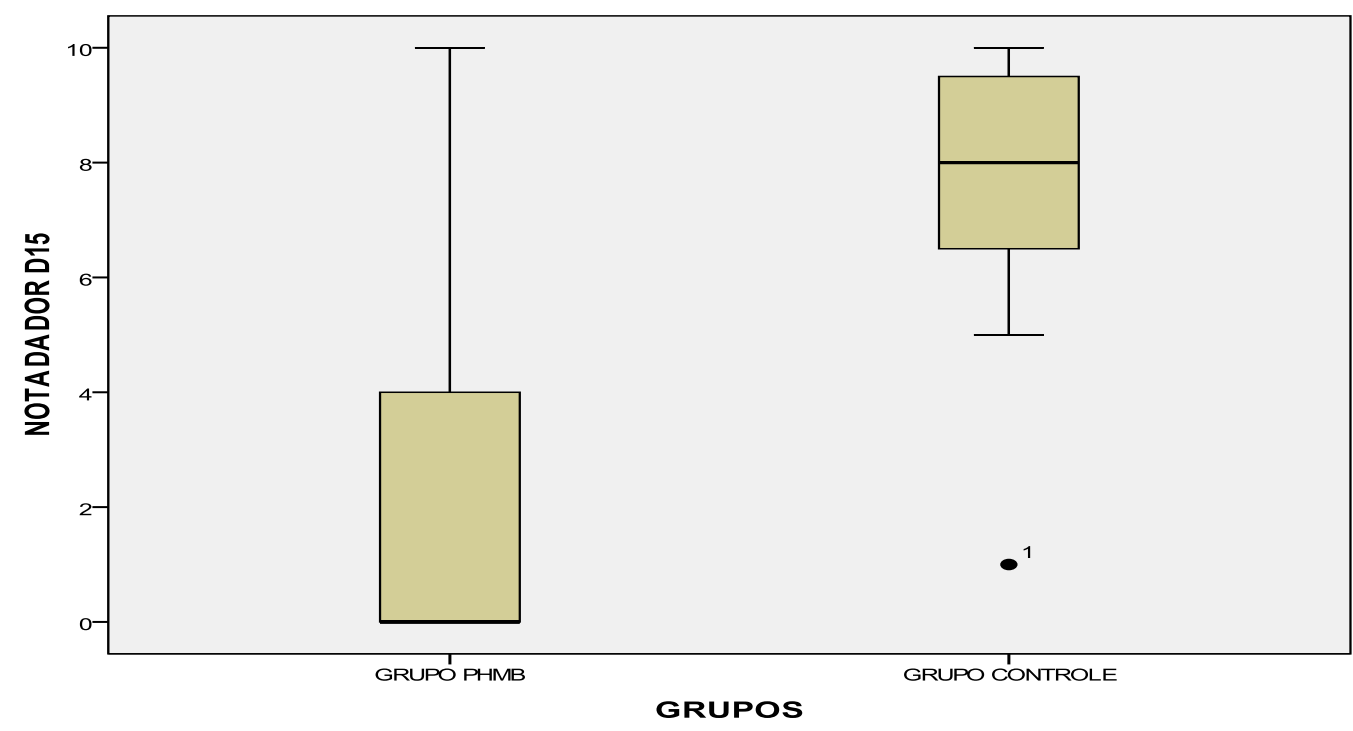

Figura 3. Distribuição da variação da média de nota de dor no D15 ( $15^{\circ}$ dia de uso do gel), segundo os grupos PHMB e controle.

\section{REFERÊNCIAS BIBLIOGRÁFICAS E OUTRAS:}

1. INSTITUTO NACIONAL DO CÂNCER. Coordenação e Prevenção e Vigilância Estimativa 2014: Incidência de Câncer no Brasil / Instituto Nacional de Câncer José Alencar Gomes da Silva, Coordenação de Prevenção e Vigilância. Rio de Janeiro: INCA, 2014. 124p. : il. col., mapas. Disponível em:< http://www.inca.gov.br/estimativa/2014/estimativa-24042014.pdf>. Acesso em 26 set 2014.

2. BRENER, S. et al. Carcinoma de células escamosas bucal: uma revisão de literatura entre o perfil do paciente, estadiamento clínico e tratamento proposto. Revista

Brasileira de Cancerologia, v. 53, n. 1, p. 63-69, 2007. 
3. CARVALHO, S. H. G.; SOARES, M.S.M.; FIGUEIREDO, R.L.Q. Levantamento Epidemiológico dos Casos 6 de Câncer de Boca em um Hospital de Referência em Campina Grande, Paraíba, Brasil. Pesq Bras Odontoped Clin Integr, João Pessoa, v.12, n.1, p.47-51, jan./mar., 2012.

4. CÂNCER no brasil: presente e futuro. Rev. Assoc. Med. Bras., Editorial, São Paulo, v. 50, n. 1, 2004 . Disponível em: . Acesso em 22 out. 2014.

5. FREITAS et al, 2009; FREITAS, D. A. et al. Sequelas bucais da radioterapia de cabeça e pescoço. Rev. CEFAC [online]., v.13, n.6, p. 1103-1108, 2011. ISSN 19820216. Disponível em:< http://www.scielo.br/pdf/rcefac/v13n6/161-10.pdf $>$. Acesso em 22 set 2014.

6. HOLMES, T. S. V. et al. Fatores relacionados ao surgimento e gradação da mucosite oral radioinduzida. Rev Cubana Estomatol [online]., v.51, n.1, p. 71-79, 2014. ISSN 0034-7507.

7. VOLPATO, L. E. R. et al. Mucosite bucal rádio e quimioinduzida. Rev. Bras. Otorrinolaringol. [online]., v.73, n.4, p. 562-568, 2007. ISSN 0034-7299. Disponível em:< http://www.scielo.br/pdf/rboto/v73n4/a17v73n4.pdf >. Acesso em 26 Set 2014.

8. LOPES, C. O; MAS, J. R. I; ZANGARO, R. A. Prevenção da xerostomia e da mucosite oral induzidas por radioterapia com uso do laser de baixa potência. Radiol Bras [online]., v.39, n.2, p. 131-136, 2006. ISSN 0100-3984. Disponível em: <http://www.scielo.br/pdf/rb/v39n2/29196.pdf>. Acesso em 26 set 2014.

9. SAWADA, N.O. et al. Quality of life evaluation in cancer patients to submitted to chemotherapy. Rev Esc Enferm USP [Internet], v.43, n. 3, p. 581-7, 2009. Disponível em. Acesso em 18 out. 2014.

10. SANTOS, R. C. S. et al. Mucosite em pacientes portadores de câncer de cabeça e pescoço submetidos à radioquimioterapia. Rev. esc. enferm. USP [online], v.45, n.6, p. 1338-1344, 2011. ISSN 0080-6234. Disponível em: http://www.scielo.br/pdf/reeusp/v45n6/v45n6a09.pdf> Acesso em 26 set 2014.

11. SONIS, S. T. et al., Perspectives on cancer therapy-induced mucosal injury: pathogenesis, measurement, epidemiology, and consequences for patients. Cancer, v. 100, n.9, p.1995-2025, 2004. Disponível em: http://onlinelibrary.wiley.com/doi/10.1002/cncr.20162/pdf>. Acesso em 22 out. 2014. 12. FIGUEIREDO, A. L. P. et al. Laser terapia no controle da mucosite oral: um estudo de metanálise. Rev. Assoc. Med. Bras. [online]., v.59, n.5, p. 467-474, 2013. ISSN 0104-4230. Disponível em:< http://www.scielo.br/pdf/ramb/v59n5/v59n5a12.pdf> Acesso em 26 set. 2014.

13. SCHIRMER, E. M.; FERRARI, A.; TRINDADE, L. C. T. Evolução da mucosite oral após intervenção nutricional em pacientes oncológicos no serviço de cuidados paliativos. Rev. dor [online], v.13, n.2, p. 141-146, 2012. ISSN 1806-0013. http://www.scielo.br/pdf/rdor/v13n2/09.pdf>. Acesso em 26 set 2014.

14. DISSEMOND, J. et al. Classification of Wounds at Risk and Their Antimicrobial Treatment with Polihexanide: A Practice-Oriented Expert Recommendation. Skin Pharmacol Physiol., v. 24, n. 5, p. 7 245-55, abr. 2011. DOI: 10.1159/000327210. PMID: 21508658 [PubMed - indexed for MEDLINE].

15. MARTINEZ , J. E.; GRASSI, D. C.; MARQUES, L. G. Análise da aplicabilidade de três instrumentos de avaliação de dor em distintas unidades de atendimento: ambulatório, enfermaria e urgência. Rev Bras Reumatol, v.51, n.4, p.299-308, 2011. Disponível em: http://www.scielo.br/pdf/rbr/v51n4/v51n4a02.pdf $>$. Acesso em 22 Out.2014. 